\title{
Marine Derived Hamacanthins as Lead for the Development of Novel PDGFRß Protein Kinase Inhibitors
}

\section{Boris Pinchuk ${ }^{1,2}$, Eugen Johannes ${ }^{1}$, Sheraz Gul ${ }^{2}$, Joachim Schlosser ${ }^{1}$, Christoph Schaechtele ${ }^{3}$, Frank Totzke ${ }^{3}$ and Christian Peifer ${ }^{1, *}$}

1 Institute of Pharmacy, University of Kiel, Gutenbergstraße 76, Kiel D-24118, Germany;

E-Mails: bpinchuk@pharmazie.uni-kiel.de (B.P.); ejohannes@pharmazie.uni-kiel.de (E.J.);

jschlosser@pharmazie.uni-kiel.de (J.S.)

2 European ScreeningPort GmbH, Schnackenburgallee 114, Hamburg D-22525, Germany;

E-Mail: sheraz.gul@screeningport.com

3 ProQinase GmbH, Breisacherstraße 117, Freiburg D-79106, Germany;

E-Mails: c.schaechtele@proqinase.com (C.S.); f.totzke@proqinase.com (F.T.)

* Author to whom correspondence should be addressed; E-Mail: cpeifer@pharmazie.uni-kiel.de; Tel.: +49-431-880-1137; Fax +49-431-880-1352.

Received: 7 June 2013; in revised form: 1 August 2013 / Accepted: 8 August 2013 /

Published: 26 August 2013

\begin{abstract}
In this study, we report on pyrazin-2(1H)-ones as lead for the development of potent adenosine triphosphate (ATP) competitive protein kinase inhibitors with implications as anti-cancer drugs. Initially, we identified the pyrazin-2(1H)-one scaffold from hamacanthins (deep sea marine sponge alkaloids) by Molecular Modeling studies as core binding motif in the ATP pocket of receptor tyrosine kinases (RTK), which are validated drug targets for the treatment of various neoplastic diseases. Structure-based design studies on a human RTK member PDGFR (platelet-derived growth factor receptor) suggested a straight forward lead optimization strategy. Accordingly, we focused on a Medicinal Chemistry project to develop pyrazin-2(1H)-ones as optimized PDGFR binders. In order to reveal Structure-Activity-Relationships (SAR), we established a flexible synthetic route via microwave mediated ring closure to asymmetric 3,5-substituted pyrazin-2(1H)-ones and produced a set of novel compounds. Herein, we identified highly potent PDGFR binders with $\mathrm{IC}_{50}$ values in an enzymatic assay below $\mu \mathrm{M}$ range, and possessing significant activity against PDGFR dependent cancer cells. Thus, marine hamacanthin-derived pyrazin-2(1H)-ones showing interesting properties as lead for their further development towards potent PDGFR-inhibitors.
\end{abstract}


Keywords: marine sponge derived hamacanthins; pyrazin-2(1H)-ones; receptor tyrosine kinases; PDGFR inhibitors; anti-cancer activity

\section{Introduction}

Marine-derived bioactive compounds and their novel chemical scaffolds have been shown to be attractive starting points for drug discovery programs [1-3]. In this regard, we became interested in the marine alkaloid family of hamacanthins [4-6]. In the course of our work to develop ATP-competitive receptor tyrosine kinase (RTK) inhibitors with anti-cancer activity [7-10], we focused on the deep-sea sponge derived bis-indole alkaloids possessing a 3,5-bis-indole-piperazin-2-one and 3,5-bis-indole-3, 4-dihydropyrazin-2(1H)-one scaffold, respectively (Figure 1) [4,5,11,12]. Recently, cis-3,4-dihydro hamacanthin B was reported to be a potent bacterial methicillin-resistant Staphylococcus protein kinase (PK) inhibitor (MRSA-PK inhibitor) with an $\mathrm{IC}_{50}$ value of $0.016 \mu \mathrm{M}$ and significant selectivity over human protein kinase isoforms [13]. Furthermore, the 2(1H)-pyrazinone scaffold is present as core moiety in PK inhibitors [14]. Among human PK are validated drug targets in oncology; over-activated RTK including VEGFR, PDGFR and c-kit are considered to be major targets for the development of clinically effective inhibitors [15-17]. In line with this notion, many anti-cancer compounds that are advanced into the clinic show (group-) selectivity towards VEGFR, FGFR, EGFR, PDGFR $\alpha / \beta$, c-kit, and Flt-3 [18]. Since all PKs from the kinome use ATP as a cofactor for the phosphorylation of proteins in signal transduction pathways, they share a highly conserved ATP binding pocket that is the molecular binding site of most PK inhibitors [19]. Thus, small molecular differences in amino acid identities adjacent to the ATP pocket provide selectivity filters for specific inhibitors [20]. Therefore, we hypothesize that the hamacanthin-core could serve as a suitable scaffold for the design of specific PK inhibitors.

Figure 1. Chemical 2D-representation and energy minimized (Schrödinger Suite 2011: MacroModel, version 9.9, Schrödinger, LLC, New York, NY, 2012) 3D-structures of hamacanthin family members, and 3,5-bis(6-bromo-1H-indol-3-yl)-1H-pyrazin-2-one as the concept of this study. The compounds formally show different oxidation status of the piperazine-2-one core with significant impact to 3,5-diaryl-conformational parameters.

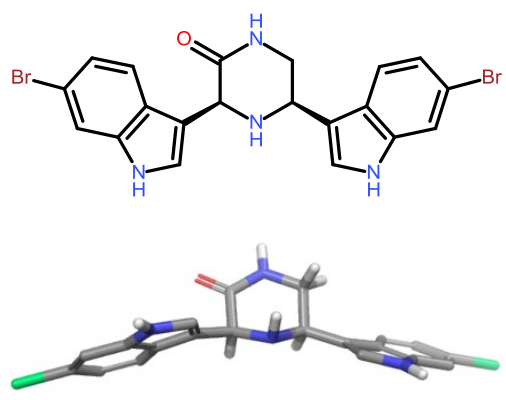

cis-3,4-dihydrohamacanthin B

(A)
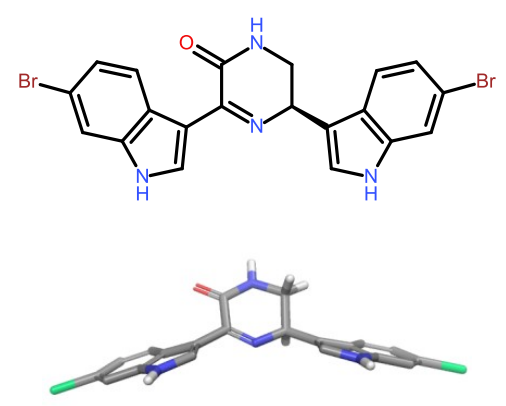

5(R)-hamacanthin $\mathrm{B}$

(B)
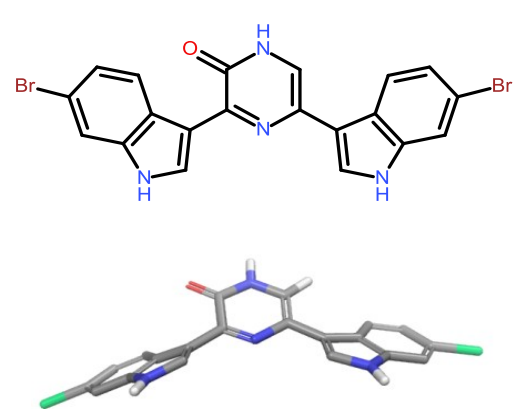

designed 3,5-diaryl-pyrazin-2(1H)-one core $(\mathbf{C})$ 
Our molecular modeling and docking approaches for the ATP binding pocket of the RTK VEGF-R2 suggests that only the 3,5-diaryl-pyrazin-2(1H)-one core (Figure 1C) is capable of occupying the narrow ATP active site of this RTK. Furthermore, the pyrazin-2(1H)-one core was reported to be a key binding motif to PK [14]. In contrast, neither the 3,5-bis-indole-piperazin-2-one nor the 3,5-bis-indole-3,4-dihydropyrazin-2 $(1 H)$-one core of hamacanthins produced plausible docking poses. The lack of reasonable binding modes in our modeling studies is mainly due to the chiral center(s) of the core scaffold positioning the 3,5-bis-indole-moieties out of the piperazin-2-one ring-plane, and the 5-indole moiety out of the 5,6-dihydropyrazin-2(1H)-one ring plane, respectively (Figure 1A,B). Our observation is in line with the notion that cis-3,4-dihydrohamacanthin B was reported to be an allosteric MRSA-PK inhibitor that addresses a tetrameric interface region of the PK-protein, and was not determined by X-ray analysis as ligand binding to the ATP site [4].

In this study, we designed the aryl-substitution pattern of the 6-membered 3,5-diaryl-pyrazin-2 (1H)-one (compound 5) based on the corresponding 5-membered 3,4-diaryl-2 $H$-pyrrole-2-one (compound 1, Figure 2), a potent inhibitor developed in a former project showing strong activity against the RTK VEGF-R2/3 $\left(\mathrm{IC}_{50}=0.03 \mu \mathrm{M}\right)$ and with good efficacy in cellular assays [9].

Figure 2. Structures of the reported potent VEGF-R2/3 inhibitor, compound $\mathbf{1}$ and the newly designed and synthesized compound $\mathbf{5}$ of this study showing comparable decoration patterns of the aryl moieties.

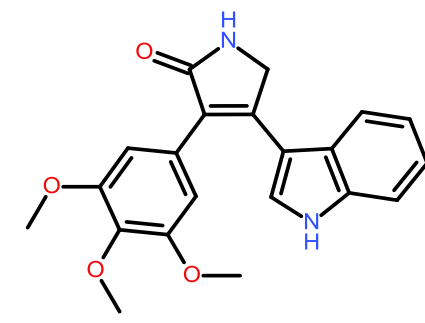

3,4-diaryl-2H-pyrrole-2-one (1)

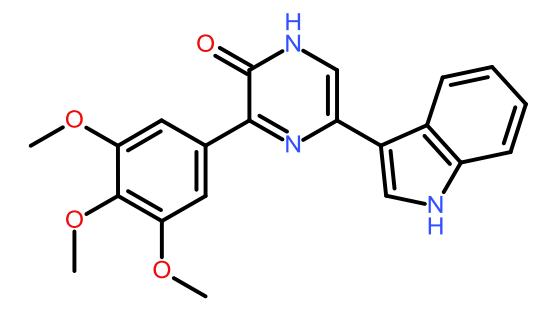

3,5-diaryl-pyrazin-2(1H)-one (5)

\section{Results and Discussion}

\subsection{Synthesis of Designed Compound 5}

In order to confirm the hypothesis that the pyrazin-2(1H)-ones are suitable scaffolds for PK inhibitor development, we synthesized compound $\mathbf{5}$ that involved a modified microwave-mediated ring closure strategy (Figure 3) [12]. In summary, glyoxylic acid 2 was activated by carbonyldiimidazole (CDI) and coupled with tryptamine to produce compound 3, which upon DDQ-oxidation yielded compound 4. Ring closure in the final step to produce the targeted pyrazin-2(1H)-one compound $\mathbf{5}$ was straightforward and involved an optimized microwave-mediated reaction using ammonium acetate as the nitrogen source.

\subsection{Biological Evaluation: Activity against PKs}

Interestingly, in a preliminary screen [21] involving 24 therapeutically relevant PK targets, compound 5 inhibited VEGF-R2/3 in the low $\mu \mathrm{M}$ range, and most potently inhibited PDGFR $\beta$ with an 
$\mathrm{IC}_{50}$ of $0.5 \mu \mathrm{M}$. Moreover, compound 5 was shown to exhibit promising selectivity over the other PK enzymes tested as part of the panel (Table 1). Thus, in order to further enhance potency of this lead compound towards the PDGFR $\beta$ drug target [22] we used a structure-based optimization approach. As no X-ray structure of the PK domain of PDGFR was available in the public domain, we generated a homology model of PDGFR $\beta$ based on the highly related RTK VEGF-R2 as template structure (pdb code 2p2h [23] using Schrödinger Prime, version 3.1, Schrödinger, LLC, New York, NY, USA, 2012).

Figure 3. Synthesis of designed compound 5 by microwave mediated ring closure strategy. Reagents and conditions: (a) 1. CDI, dichloromethane 2. tryptamine; (b) DDQ, THF; (c) $\mathrm{NH}_{4} \mathrm{Ac}, \mathrm{HAc}$, microwave heating for $5 \mathrm{~min}$.

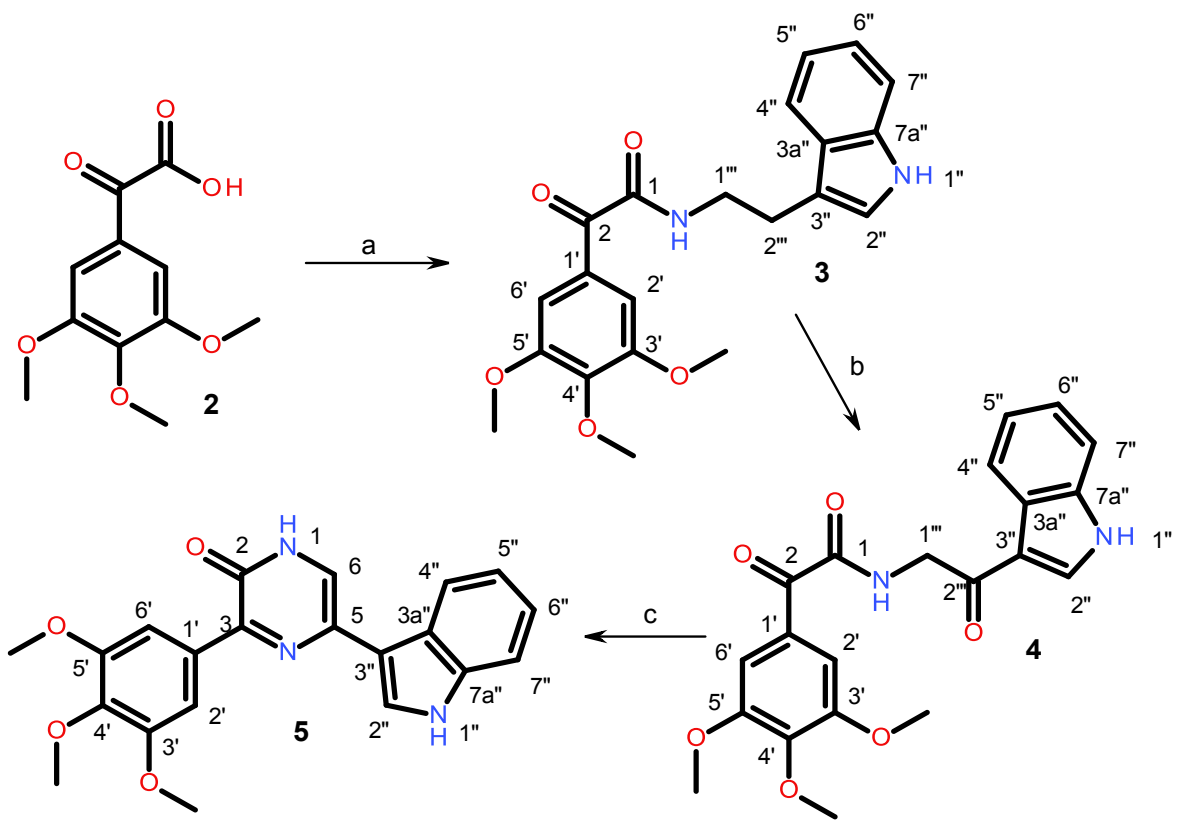

Table 1. $\mathrm{IC}_{50}$ values $(\mu \mathrm{M})$ of compounds 1 and 5 in a panel [21] of 24 therapeutically relevant PK targets (- indicates no significant PK inhibition was observed at a compound concentration of $100 \mu \mathrm{M})$.

\begin{tabular}{ccc}
\hline Protein kinase & $\mathbf{I C}_{\mathbf{5 0}}(\boldsymbol{\mu M})$ for Compound $\mathbf{1}$ & $\mathbf{I C}_{\mathbf{5 0}}(\boldsymbol{\mu M})$ for Compound 5 \\
\hline AKT1 & - & - \\
ARK5 & 16 & - \\
Aurora-A & 46 & - \\
Aurora-B & 66 & - \\
B-RAF-VE & - & - \\
CDK2/CycA & 66 & - \\
CDK4/CycD1 & 56 & - \\
COT & 32 & - \\
EGFR & 23 & - \\
EPHB4 & 53 & - \\
ERBB2 & 51 & - \\
FAK & 9 & - \\
IGF1R & 22 & - \\
SRC & 14 & - \\
\hline
\end{tabular}


Table 1. Cont.

\begin{tabular}{ccc}
\hline VEGF-R2 & 0.031 & 4 \\
VEGF-R3 & 0.037 & 5 \\
FLT3 & 61 & 36 \\
INSR & 43 & - \\
MET & 60 & - \\
PDGFR $\beta$ & 11 & 0.5 \\
PLK1 & - & - \\
SAK & 10 & - \\
TIE2 & 5 & - \\
CK2a1 & - & - \\
\hline
\end{tabular}

\subsection{Molecular Modeling}

Docking compound 5 into the ATP binding site of our homology model of PDGFR $\beta$ revealed an interesting and reasonable binding mode (Figure 4, details of the methodology can be found in Section 3.2). The key features of the interaction of compound 5 include the hydrogen bonds formed with the backbone carbonyl of Glu101 and to the amine of Cys103 in the hinge region of the PK, respectively. The indole ring is located in the hydrophobic pocket I whereas the trimethoxyphenyl moiety is located in the solvent exposed hydrophobic region II (in accord with a type-I PK inhibitor [24]).

Figure 4. (A) Homology model of PDGFR $\beta$ based on template structure of VEGF-R2 (pdb code $2 \mathrm{p} 2 \mathrm{~h}$ [23]) and modeled binding mode of compound $\mathbf{5}$ in the ATP pocket. (B) Ligand-interaction diagram of compound $\mathbf{5}$ in the ATP binding site of the PDGFR $\beta$ homology model. Key amino acid residues and hydrogen bonds are shown (see legend).

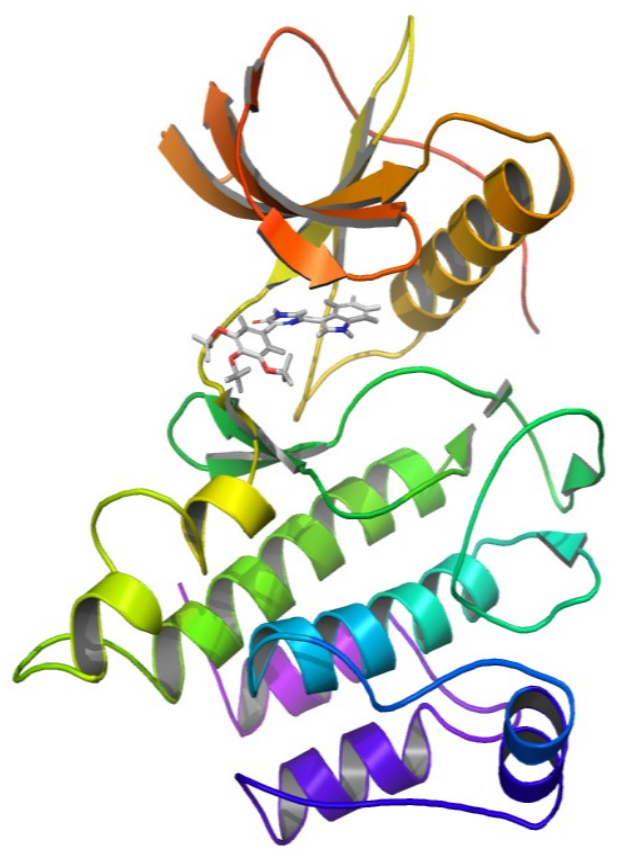

(A)

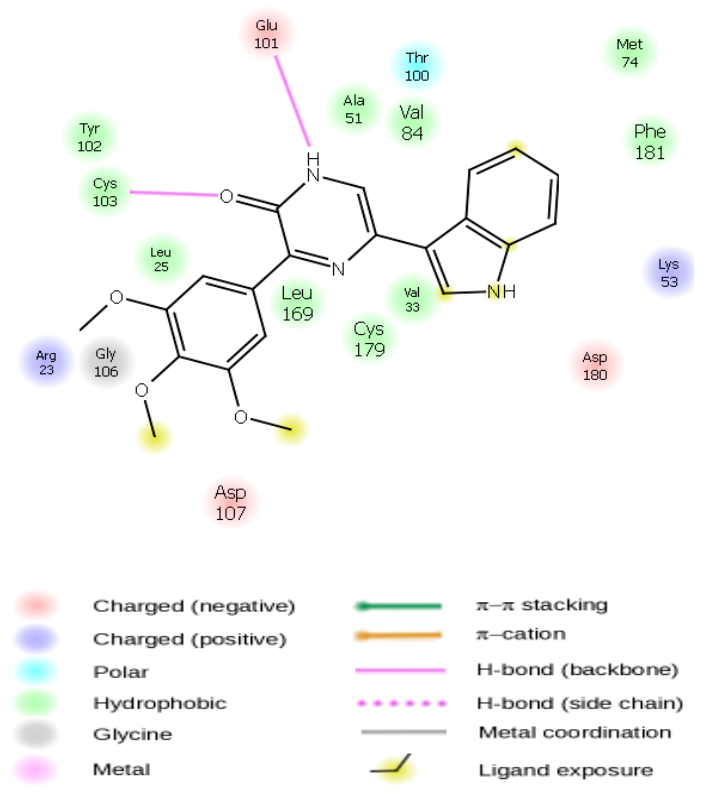

(B) 
Having demonstrated a rational binding mode for compound $\mathbf{5}$ in the active site of PDGFR $\beta$, we subsequently aimed to optimize this compound by performing a virtual screen against a focused set of compounds, with particular focus upon varying the indole at the pyrazin-2(1H)-one $5^{\prime}$ position, as the pyrazin-2(1H)-one 3'-(3,4,5-trimethoxyphenyl) moiety had already been shown to be required in order to inhibit PDGFR $\beta$ (Figure $5[9,25]$ ).

Figure 5. Aryl variation of the pyrazin-2(1H)-one 3'-position and PDGFR $\beta$ IC $_{50}$ values $(\mu \mathrm{M})$ of compounds 5-7.
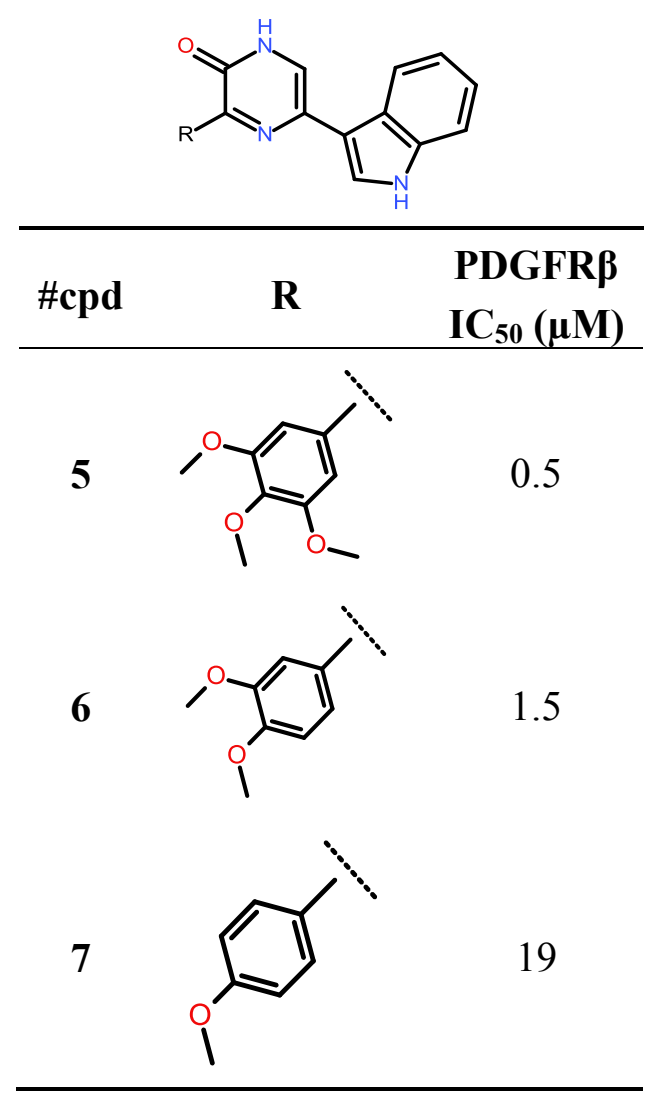

Figure 6. 5'OH-Indole variation and PDGFR $\beta-\mathrm{IC}_{50}$ values $(\mu \mathrm{M})$ of compounds 5 and $\mathbf{8}$.

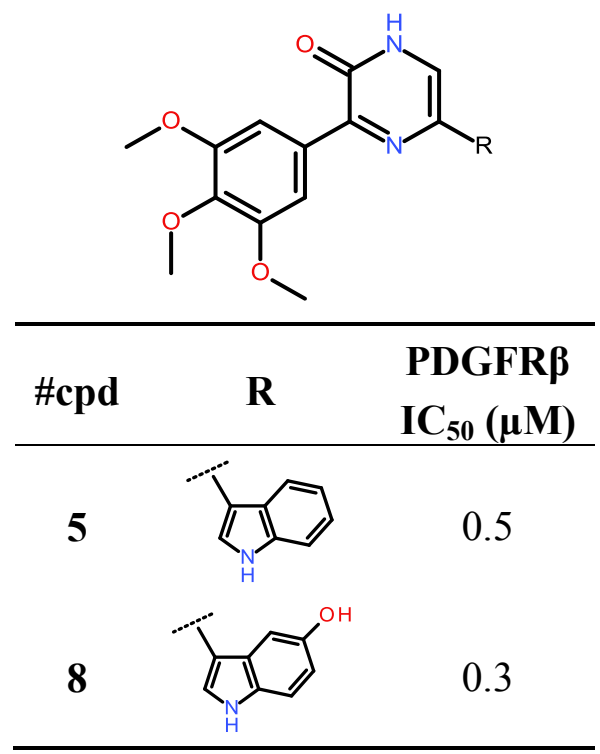


Thus, the indole moiety originally present in the pyrazin-2(1H)-one 5'-position was systematically varied by synthetically feasible decoration patterns yielding a set of virtual compounds. The subsequent ligand preparation, docking and scoring campaign using Schrödinger Glide SP (Suite 2012 Glide version 5.8, Schrödinger, LLC, New York, NY, USA) produced a list with compound $\mathbf{8}$ as the one that was predicted to inhibit PDGFR $\beta$ most potently (Figure 6). The general binding mode of compound $\mathbf{8}$ is comparable to the binding pose of compound 5 with the pyrazin-2(1H)-one core addressing H-bonds to Glu101 and Cys103 (Figure 4), but with the indole-5'OH situated in the hydrophobic pocket II addressing an additional H-bond to the backbone amide-carbonyl oxygen of Val84.

In accordance with our modeling, compound $\mathbf{8}$ showed slightly enhanced potency against PDGFR $\beta$ in the PDGFR $\beta$ assay $\left(\mathrm{IC}_{50}\right.$ value $=0.3 \mu \mathrm{M}$, Figure 6$)$.

\subsection{Biological Activity in Cancer Cell Lines of Compounds $\mathbf{5}$ and $\mathbf{8}$}

In order to determine the cytotoxic profiles of compounds $\mathbf{5}$ and $\mathbf{8}$, they were evaluated in cell viability assays (Figure 7) including HL-60, a human myeloblastic leukemia cell line, as it has been shown that the proliferation and differentiation of these cells depend upon PDGFR-signalling [26]. Cells were treated with compounds $\mathbf{5}$ and $\mathbf{8}$ and after $48 \mathrm{~h}$ incubation, their viability was determined. The compounds exhibited a differentiated cytotoxic profile. Both compounds were shown to be cytotoxic against HL-60 cells such that compound 5 was associated with an $\mathrm{IC}_{50}$ value of $0.026 \mu \mathrm{M}$ and exhibited a significantly stronger anti-proliferative effect than compound $\mathbf{8}$ that had an $\mathrm{IC}_{50}$ value of $30 \mu \mathrm{M}$. This is in sharp contrast to the data from isolated PDGFR $\beta$ assay and may be due to limited cellular bioavailability of $\mathbf{8}$. Interestingly, the other cells tested were significantly less affected. This is in line with the notion that HL-60 cells depend on PDGFR-signaling [26]. However, in our ongoing studies we are currently investigating further molecular details of the reported selectivity of compound $\mathbf{5}$ against HL-60 cells.

Figure 7. Determination of cytotoxic profiles of compounds $\mathbf{5}$ and $\mathbf{8}$ in cell viability assays using HL-60, TK 10, 786-0, M 14, and MCF-7 cell lines.
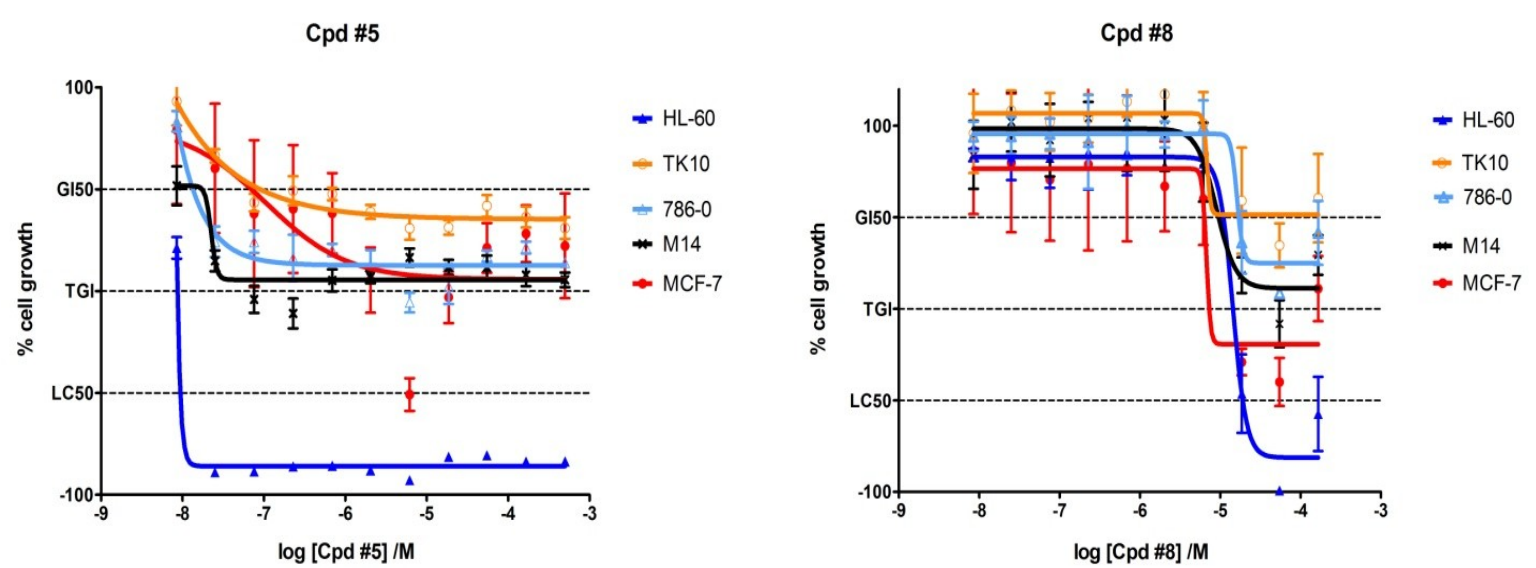


\section{Experimental Section}

\subsection{Chemistry and Synthesis of Test Compounds}

${ }^{1} \mathrm{H}(300 \mathrm{MHz})$ and ${ }^{13} \mathrm{C}(75 \mathrm{MHz}) \mathrm{NMR}$ were recorded on a Bruker Avance III 300 spectrometer (Rheinstetten, Germany) at $300 \mathrm{~K}$ with a multinuclear probe head using the manufacturer's pulse programs. The data are reported as follows: chemical shift in ppm from $\mathrm{Me}_{4} \mathrm{Si}$ (TMS) as external standard, multiplicity and coupling constant $(\mathrm{Hz})$. NMR spectra were obtained on a ${ }^{1} \mathrm{H}(300 \mathrm{MHz})$ and ${ }^{13} \mathrm{C}$ spectra $(75 \mathrm{MHz})$ were referenced either to TMS or to internal DMSO- $\mathrm{d}_{5}\left({ }^{1} \mathrm{H} \mathrm{NMR} \delta 2.50\right)$ and internal DMSO- $\mathrm{d}_{6}\left({ }^{13} \mathrm{C} \mathrm{NMR} \delta 39.5\right)$ or internal $\mathrm{CHCl}_{3}\left({ }^{1} \mathrm{H} \mathrm{NMR} \delta 7.26\right)$ and internal $\mathrm{CDCl}_{3}$ $\left({ }^{13} \mathrm{C}\right.$ NMR $\delta$ 77.0). All coupling constants ( $J$ values) are quoted in Hz. The following NMR abbreviations are used: br (broad), s (singlet), d (doublet), $\mathrm{t}$ (triplet), m (unresolved multiplet). The labelling scheme of structures to correlate NMR signals can be found in Supporting Information.

Mass spectra of the compounds were recorded after chromatographic separation. Mixtures were separated with an Agilent 1100 HPLC system (Waldbronn; Germany) consisting of a thermostated autosampler, diode array detection and an Agilent Zorbax Eclipse XDB-C8 column $(150 \times 4.6 \mathrm{~mm}$, $5 \mu \mathrm{m}$ particle size). Elution was achieved with a solvent gradient system of water and acetonitrile, with $0.1 \%$ of acetic acid and a flow rate of $1 \mathrm{~mL} / \mathrm{min}$. The eluent flow was splitted to the mass spectrometer.

Mass spectra with nominal resolution were recorded with an Esquire $\sim$ LC mass spectrometer (Bruker Daltonik, Bremen, Germany), with electrospray ionization operating in the positive ion mode, with the following parameters: drying gas nitrogen $8 \mathrm{~L} / \mathrm{min}$, nebulizer $35 \mathrm{psi}$, dry gas heating $350{ }^{\circ} \mathrm{C}$, HV capillary $4000 \mathrm{~V}$, HV EndPlate offset $-500 \mathrm{~V}$. GC/MS was performed on a HP6890 Series System. EI-Mass spectra were recorded on a Varian MAT 311A (70 eV). HRMS spectra were recorded on a MAT-95 (Finnigan).

Melting points/decomposition temperatures were determined on a Büchi apparatus according to Dr. Tottoli and are uncorrected.

Where appropriate, column chromatography was performed for crude precursors with Merck silica gel $60(0.063-0.200 \mathrm{~mm})$ or Acros organics silica gel $(0.060-0.200 \mathrm{~mm}$; pore diameter $c a .60 \mathrm{~nm})$. Column chromatography for test compounds was performed using a La-Flash-System (VWR) with Merck silica gel $60(0.015-0.040 \mathrm{~mm})$ or RP8 columns. The progress of the reactions was monitored by thin-layer chromatography (TLC) performed with Merck silica gel 60 F-245 plates. Where necessary, reactions were carried out in a nitrogen atmosphere using $4 \AA$ molecular sieves. All reagents and solvents were obtained from commercial sources and used as received (THF was used after distillation over K/benzophenone). Reagents were purchased from Sigma-Aldrich Chemie, Steinheim, Germany; Lancaster Synthesis, Mühlheim, Germany or Acros, Nidderau, Germany.

HPLC analysis was performed on a Hewlett-Packard HP 1090 Series II using a Thermo Betasil C8 $(150 \times 4.6,5 \mu \mathrm{M})$ column (mobile phase flow $1.5 \mathrm{~mL} / \mathrm{min}$, gradient $\mathrm{KH}_{2} \mathrm{PO}_{4}$ buffer $\mathrm{pH} 2.3 / \mathrm{methanol}$, UV-detection $230 / 254 \mathrm{~nm}$ ). All key compounds were proven by this method to show $\geq 98 \%$ purity.

\subsubsection{Synthesis of Compound 3}

CDI (1.1 equivalent) was added to a solution of 1 equivalent 2-oxo-2-(3,4,5-trimethoxyphenyl)acetic acid (2) in $N$-methylpyrrolidone and reacted at room temperature for $1 \mathrm{~h}$. Then, tryptamine (1 equiv) 
was added and the mixture was stirred overnight. Then, water was added to quench the reaction and the mixture was extracted three times with EtOAc. The combined organic phase was evaporated under reduced pressure and the residue purified by flash silica gel chromatography to afford $N$-[2-(1H-indol-3-yl)ethyl]-2-oxo-2-(3,4,5-trimethoxyphenyl)acetamide (3). Yield: (2.2 g, 91\%); mp $119{ }^{\circ} \mathrm{C} ;{ }^{1} \mathrm{H}$ NMR (300 MHz, DMSO-d $\left.d_{6}\right) \delta: 2.98$ (t, $\left.J=7.3 \mathrm{~Hz}, 2 \mathrm{H}, \mathrm{CH}_{2}-2^{\prime \prime \prime}\right), 3.57$ (dt, $J=7.1,6.1 \mathrm{~Hz}$, $\left.2 \mathrm{H}, \mathrm{CH}_{2}-1^{\prime \prime \prime}\right), 3.77$ (s, 9H, $3 \mathrm{OMe}$ ), 6,98 (t, $\left.J=6.9 \mathrm{~Hz}, 1 \mathrm{H}, \mathrm{H}-55^{\prime \prime}\right), 7.07$ (t, $\left.J=7.0 \mathrm{~Hz}, 1 \mathrm{H}, \mathrm{H}-6^{\prime \prime}\right), 7.20$ (d, $\left.J=2.3 \mathrm{~Hz}, 1 \mathrm{H}, \mathrm{H}-2^{\prime \prime}\right), 7.28$ (s, 2H, H-2',6'), 7.34 (d, $\left.J=8.0 \mathrm{~Hz}, 1 \mathrm{H}, \mathrm{H}-7^{\prime \prime}\right), 7.57$ (d, $J=7.7 \mathrm{~Hz}, 1 \mathrm{H}$, H-4"), 8.99 (t, $J=5.75 \mathrm{~Hz}, 1 \mathrm{H}, \mathrm{CONH}), 10.82$ (s, 1H, NH-1"); ${ }^{13} \mathrm{C}$ NMR (75 MHz, DMSO- $\left.d_{6}\right) \delta: 24.8$ $\left(\mathrm{CH}_{2}-2^{\prime \prime \prime}\right), 39.1\left(\mathrm{CH}_{2}-1^{\prime \prime \prime}\right), 56.0$ (2',5'-OMe), 60.3 (4'-OMe), 107.3 (CH-2',6'), 111.3 (CH-7"), 111.4 $\left(\mathrm{C}_{\mathrm{q}}-3^{\prime \prime}\right), 118.2\left(\mathrm{CH}-5^{\prime \prime}, 4^{\prime \prime}\right), 120.9\left(\mathrm{CH}-6^{\prime \prime}\right), 122.7\left(\mathrm{CH}-2^{\prime \prime}\right), 127.1\left(\mathrm{C}_{\mathrm{q}}-3 \mathrm{a}^{\prime \prime}\right), 128.0\left(\mathrm{C}_{\mathrm{q}}-1^{\prime}\right), 136.2\left(\mathrm{C}_{\mathrm{q}}-7 \mathrm{a}^{\prime \prime}\right)$, $143.1\left(\mathrm{C}_{\mathrm{q}}-4^{\prime}\right), 152.8\left(\mathrm{C}_{\mathrm{q}^{-}} 3^{\prime}, 5^{\prime}\right), 164.6(\mathrm{CO}-1), 188.9(\mathrm{CO}-2)$; LC-MS $m / z 383[\mathrm{M}+\mathrm{H}]^{+}$.

\subsubsection{Synthesis of Compound 4}

To a solution of 3 in THF/ $\mathrm{H}_{2} \mathrm{O}(9: 1)$ at $0{ }^{\circ} \mathrm{C}$, DDQ (1.5 equiv. dissolved in THF) was added dropwise and stirred for $1 \mathrm{~h}$. Then the solvent was evaporated to dryness. To the residual mixture, methanol was added. The precipitate was filtered off and washed with $\mathrm{H}_{2} \mathrm{O}$ and methanol to afford $N$-[2-(1H-indol-3-yl)-2-oxo-ethyl]-2-oxo-2-(3,4,5-trimethoxyphenyl)acetamide (4). Yield: (363 mg, 58\%); mp $201{ }^{\circ} \mathrm{C}$; ${ }^{1} \mathrm{H}$ NMR (300 MHz, DMSO- $\left.d_{6}\right) \delta: 3.80$ (s, 3H, OMe), 3.93 (s, 6H, $2 \mathrm{OMe}$ ), 4.69 (d, $\left.J=6.0 \mathrm{~Hz}, 2 \mathrm{H}, \mathrm{CH}_{2}-1^{\prime \prime}\right), 7.23$ (m, 2H, H-5",6"), 7.51 (m, 1H, H-7"), 7.57 (s, 2H, H-2',6'), 8.16 (m, 1H, H-4"), 8.51 (d, $\left.J=3.15 \mathrm{~Hz}, 1 \mathrm{H}, \mathrm{H}-2^{\prime \prime}\right), 9.21$ (t, $\left.J=5.9 \mathrm{~Hz}, 1 \mathrm{H}, \mathrm{CONH}\right), 12.08$ (s, $\left.1 \mathrm{H}, \mathrm{NH}-1^{\prime \prime}\right) ;{ }^{13} \mathrm{C}$ NMR (75 MHz, DMSO- $\left.d_{6}\right) \delta$ : $45.4\left(\mathrm{CH}_{2}-1^{\prime \prime \prime}\right), 56.1$ (2',5'-OMe), 60.3(4'-OMe), 107.5 (CH-2',6'), 112.2 $\left(\mathrm{CH}-7^{\prime \prime}\right), 113.8\left(\mathrm{C}_{\mathrm{q}^{-}} 3^{\prime \prime}\right), 121.0\left(\mathrm{CH}-4^{\prime \prime}\right), 122.0,122.9\left(\mathrm{CH}-5^{\prime \prime}, 6^{\prime \prime}\right), 125.4\left(\mathrm{C}_{\mathrm{q}}-3 \mathrm{a}^{\prime \prime}\right), 128.2\left(\mathrm{C}_{\mathrm{q}}-1^{\prime}\right), 133.9$ $\left(\mathrm{CH}-2^{\prime \prime}\right), 136.4\left(\mathrm{C}_{\mathrm{q}}-7 \mathrm{a}^{\prime \prime}\right), 143.1\left(\mathrm{C}_{\mathrm{q}}-4^{\prime}\right), 152.9\left(\mathrm{C}_{\mathrm{q}}-3^{\prime}, 5^{\prime}\right), 166.3$ (CO-1), 189.1 (CO-2), 189.7 (CO-2'"'); LC-MS $m / z 397[\mathrm{M}+\mathrm{H}]^{+}$.

General procedure for pyrazinone ring closure using microwave synthesis (compounds $\mathbf{5}, \mathbf{6}$ and 8a) [27].

A microwave vial $(5 \mathrm{~mL})$ was equipped with ammonium acetate (10 equiv) and a solution of diketone 4 [27] ( 1 equiv) in acetic acid $\left(3 \mathrm{~mL}\right.$ ). The vial was sealed and stirred at $160{ }^{\circ} \mathrm{C}$ for 4 min in a microwave synthesizer (CEM Discover). The reaction vessel was cooled to rt when $\mathrm{H}_{2} \mathrm{O}$ was added to precipitate the pyrazinone, which was filtered off. The pyrazinone was purified by preparative HPLC (RP-phase) to afford the test compound $\geq 98 \%$ purity.

\subsubsection{Synthesis of Compound 5}

By using the general procedure for pyrazinone ring closure we obtained 5-(1H-indole-3-yl)-3-(3, 4,5-trimethoxyphenyl)pyrazin-2(1H)-one (5). Yield after final purification: (31 mg, 11\%); mp $293{ }^{\circ} \mathrm{C}$; ${ }^{1} \mathrm{H}$ NMR (300 MHz, DMSO-d $\left.d_{6}\right) \delta: 3.76$ (s, 3H, OMe), 3.88 (s, 6H, $2 \mathrm{OMe}$ ), 7.12 (m, 2H, H-5",6"), 7.44 (m, 1H, H-7"), 7.90 (s, 1H, H-6), 7.92 (d, J=2.6 Hz, 1H, H-2"), 8.01 (s, 2H, H-2',6'), 8.30 (d, $\left.J=7.4 \mathrm{~Hz}, 1 \mathrm{H}, \mathrm{H}-4^{\prime \prime}\right), 11.34$ (s, $\left.1 \mathrm{H}, \mathrm{NH}-1^{\prime \prime}\right), 12.53$ (s, 1H, NH-1); ${ }^{13} \mathrm{C}$ NMR $\left(75 \mathrm{MHz}, \mathrm{DMSO}-d_{6}\right)$

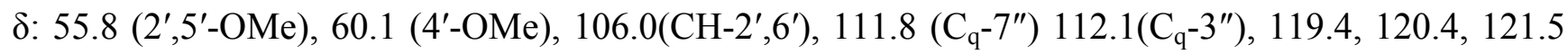
$\left(\mathrm{CH}-4^{\prime \prime}, 5^{\prime \prime}, 6^{\prime \prime}\right), 123.1\left(\mathrm{CH}-2^{\prime \prime}, 6\right), 124.8\left(\mathrm{C}_{\mathrm{q}}-3 \mathrm{a}^{\prime \prime}\right), 131.9\left(\mathrm{C}_{\mathrm{q}}-1^{\prime}, 7 \mathrm{a}^{\prime \prime}\right), 136.7\left(\mathrm{C}_{\mathrm{q}}-3,5\right), 138.9\left(\mathrm{C}_{\mathrm{q}}-4^{\prime}\right), 152.3$ 
$\left(\mathrm{C}_{\mathrm{q}}-3^{\prime}, 5^{\prime}\right), 154.1(\mathrm{CO}-2)$; LC-MS m/z $378[\mathrm{M}+\mathrm{H}]^{+}$. HRMS: $m / z$ calculated for $[\mathrm{M}]^{+} \mathrm{C}_{21} \mathrm{H}_{19} \mathrm{~N}_{3} \mathrm{O}_{4}$ : 377.1375 ; found 377.1363 .

\subsubsection{Synthesis of Compound 6}

By using general procedure for pyrazinone ring closure, 3-(3,4-dimethoxyphenyl)-5-(1H-indol-3yl)-1H-pyrazin-2-one (6) was synthesized from $N$-[2-(1H-indole-3-yl)-2-oxoethyl]-2-(3,4-dimethoxy phenyl)-2-oxoacetamide [27]. Yield: (50 mg, 51\%); mp $259{ }^{\circ} \mathrm{C} ;{ }^{1} \mathrm{H}$ NMR (300 MHz, DMSO- $\left.d_{6}\right)$ $\delta: 3.84$ (s, 3H, OMe), 3.87 (s, 3H, OMe), 7.12 (m, 3H, H-3',5",6"), 7.44 (d, J=7.4 Hz, 1H, H-7"), 7.81 (s, 1H, H-6), 7.90 (d, $\left.J=2.6 \mathrm{~Hz}, 1 \mathrm{H}, \mathrm{H}-2^{\prime \prime}\right), 8.18$ (d, $\left.J=1.9 \mathrm{~Hz}, 1 \mathrm{H}, \mathrm{H}-6^{\prime}\right), 8.25$ (d, $J=7.7 \mathrm{~Hz}, 1 \mathrm{H}$, H-4"), 8.30 (dd, $\left.J=8.6,1.9 \mathrm{~Hz}, 1 \mathrm{H}, \mathrm{H}-2^{\prime}\right), 11.34$ (s, 1H, NH-1"), 12.40 (s b, 1H, NH-1); ${ }^{13} \mathrm{C}$ NMR (75 MHz, DMSO- $\left.d_{6}\right) \delta:$ 55.3, 55.5 (2 OMe), $110.9\left(\mathrm{CH}-3^{\prime}\right), 111.4\left(\mathrm{CH}-6{ }^{\prime \prime}\right), 111.8\left(\mathrm{CH}-7^{\prime \prime}\right), 112.3$ $\left(\mathrm{C}_{\mathrm{q}^{-}} 3^{\prime \prime}\right), 119.4\left(\mathrm{CH}-6^{\prime}\right), 120.4\left(\mathrm{CH}-4^{\prime \prime}\right), 121.4\left(\mathrm{CH}-5^{\prime \prime}\right), 122.1\left(\mathrm{CH}-2^{\prime}\right), 123.1$ (CH-2",6), 124.8 $\left(\mathrm{C}_{\mathrm{q}}-3 \mathrm{a}^{\prime \prime}\right), 129.3\left(\mathrm{C}_{\mathrm{q}}-1^{\prime}\right), 136.7\left(\mathrm{C}_{\mathrm{q}}-7 \mathrm{a}^{\prime \prime}\right), 148.1\left(\mathrm{C}_{\mathrm{q}}-3^{\prime \prime}, 5^{\prime \prime}, 5^{\prime}\right), 150.2\left(\mathrm{C}_{\mathrm{q}}-4^{\prime}\right), 154.1(\mathrm{CO}-2)$; LC-MS $\mathrm{m} / \mathrm{z}$ $348[\mathrm{M}+\mathrm{H}]^{+}$. HRMS: $m / z$ calculated for $[\mathrm{M}]^{+} \mathrm{C}_{20} \mathrm{H}_{17} \mathrm{~N}_{3} \mathrm{O}_{3}: 347.1270$; found: 347.1254 .

\subsubsection{Synthesis of Compound 7}

By using general procedure for pyrazinone ring closure, 3-(4-methoxyphenyl)-5-(1H-indol-3-yl)$1 H$-pyrazin-2-one (7) was synthesized from $N$-[2-(1H-indole-3-yl)-2-oxoethyl]-2-(4-methoxyphenyl)-2oxoacetamide [27]. Yield: (200 mg, 58\%); mp $232{ }^{\circ} \mathrm{C} ;{ }^{1} \mathrm{H}$ NMR (300 MHz, DMSO- $\left.d_{6}\right) \delta: 3.84$ (s, $3 \mathrm{H}$, OMe), 7.06 (d, $\left.J=9.1 \mathrm{~Hz}, 2 \mathrm{H}, \mathrm{H}-3^{\prime}, 5^{\prime}\right), 7.10-7.18$ (m, 2H, H-5",6"), 7.43 (d, J=7.1 Hz, 1H, H-7"), 7.78 (s, 1H, H-6), 7.88 (d, $\left.J=2.6 \mathrm{~Hz}, 1 \mathrm{H}, \mathrm{H}-2^{\prime \prime}\right), 8.15$ (d, $\left.J=7.2 \mathrm{~Hz}, 1 \mathrm{H}, \mathrm{H}-4{ }^{\prime \prime}\right), 8.50$ (d, $J=9.0 \mathrm{~Hz}, 2 \mathrm{H}$, $\left.\mathrm{H}-2^{\prime}, 6^{\prime}\right), 11.34$ (s, 1H, NH-1"), 12.42 (s, 1H, NH-2); ${ }^{13} \mathrm{C}$ NMR (75 MHz, DMSO-d 6 ) $\delta: 55.2(\mathrm{OMe})$, 111.7 (CH-7"), $112.3\left(\mathrm{C}_{\mathrm{q}} \mathrm{-3}^{\prime \prime}\right), 113.4\left(\mathrm{CH}-3^{\prime}, 5^{\prime}\right), 119.5$ (CH-6"), 120.2 (CH-7"), 121.4 (CH-5"), 123.2 $\left(\mathrm{CH}-2^{\prime \prime}, 6\right), 124.7\left(\mathrm{C}_{\mathrm{q}}-3 \mathrm{a}^{\prime \prime}, 5\right), 129.1\left(\mathrm{C}_{\mathrm{q}^{-}}{ }^{\prime}\right), 130.0\left(\mathrm{CH}-2^{\prime}, 6^{\prime}\right), 136.7\left(\mathrm{C}_{\mathrm{q}}-3,7 \mathrm{a}^{\prime \prime}\right), 154.2(\mathrm{CO}-2), 160.3$ $\left(\mathrm{C}_{\mathrm{q}}-4^{\prime}\right)$; LC-MS $m / z 318[\mathrm{M}+\mathrm{H}]^{+}$. HRMS: $m / z$ calculated for $[\mathrm{M}]^{+} \mathrm{C}_{19} \mathrm{H}_{15} \mathrm{~N}_{3} \mathrm{O}_{2}$ : 317.1164; found: 317.1175 .

\subsubsection{Synthesis of Compound $\mathbf{8 a}$}

By using general procedure for pyrazinone ring closure 5-(5-benzyloxy-1H-indol-3-yl)-3-(3,4,5trimethoxyphenyl)-1H-pyrazin-2-one, compound 8a was synthesized from $N$-[2-(5-benzoxy-1H-indol3-yl)-2-oxo-ethyl]-2-oxo-2-(3,4,5-trimethoxyphenyl)acetamide [27]. Yield: (220 mg, 76\%); mp $231{ }^{\circ} \mathrm{C} ;{ }^{1} \mathrm{H}$ NMR (300 MHz, DMSO- $\left.d_{6}\right) \delta: 3.73$ (s, 3H, OMe), 3.81 (s, 6H, $\left.2 \mathrm{OMe}\right), 5.09$ (s, 2H, $\mathrm{CH}_{2}$ ), 6.93 (dd, $\left.J=8.8,2.40 \mathrm{~Hz}, 1 \mathrm{H}, \mathrm{H}-6^{\prime \prime}\right), 7.32-7.46$ (m, 6H, H-7", 5 H-Bn), 7.88 (s, 1H, H-6), 7.90 (d, $\left.J=2.5 \mathrm{~Hz}, 2 \mathrm{H}, \mathrm{H}-2^{\prime \prime}, 4^{\prime \prime}\right), 7.97$ (s, 2H, H-2',6'), 11.22 (d, $\left.J=2.5 \mathrm{~Hz}, 1 \mathrm{H}, \mathrm{NH}-1^{\prime \prime}\right), 12.36$ (s, 1H, NH-1);

${ }^{13} \mathrm{C}$ NMR (75 MHz, DMSO- $\left.d_{6}\right) \delta$ : 55.9 (2 OMe), $60.1(\mathrm{OMe}), 70.3\left(\mathrm{CH}_{2}\right), 105.4(\mathrm{CH}-4 "), 106.2$ $\left(\mathrm{CH}-2^{\prime}, 6^{\prime}\right), 111.1\left(\mathrm{CH}-6^{\prime \prime}\right), 111.7\left(\mathrm{C}_{\mathrm{q}}-3^{\prime \prime}\right), 112.1\left(\mathrm{CH}-7^{\prime \prime}\right), 124.0\left(\mathrm{CH}-2^{\prime \prime}, 6\right), 125.3\left(\mathrm{C}_{\mathrm{q}}-3 \mathrm{a}^{\prime \prime}\right), 127.7$, 127.7, 128.3 (5 CH-Bn), $132.0\left(\mathrm{C}_{\mathrm{q}}-7 \mathrm{a}^{\prime \prime}\right), 132.2\left(\mathrm{C}_{\mathrm{q}^{-}} \mathbf{1}^{\prime}, 3 \mathrm{a}^{\prime \prime}\right), 137.6\left(\mathrm{C}_{\mathrm{q}^{-}}-3,5\right), 139.0\left(\mathrm{C}_{\mathrm{q}}-4^{\prime}\right), 152.3$ $\left(\mathrm{C}_{\mathrm{q}^{-}}-3^{\prime}, 5^{\prime}\right), 152.8\left(\mathrm{C}_{\mathrm{q}}-5^{\prime \prime}\right), 154.1$ (CO-2); LC-MS $m / z 484[\mathrm{M}+\mathrm{H}]^{+}$. HRMS: $m / z$ calculated for [M] $]^{+}$ $\mathrm{C}_{28} \mathrm{H}_{25} \mathrm{~N}_{3} \mathrm{O}_{5}$ : 483.1794; found: 483.1776. 


\subsubsection{Synthesis of Compound $\mathbf{8}$}

A microwave vial $(5 \mathrm{~mL})$ was equipped with compound 8a $(100 \mathrm{mg})$, cyclohexene $(90 \mathrm{mg}), \mathrm{Pd} / \mathrm{C}$ $10 \%(20 \mathrm{mg})$ and methanol $(1.5 \mathrm{~mL})$. The vial was sealed and stirred $10 \mathrm{~min}$ at $100{ }^{\circ} \mathrm{C}$ in a microwave synthesizer. The solution was filtered and cooled to room temperature to form a precipitate which was filtered and washed with methanol and $\mathrm{Et}_{2} \mathrm{O}$ to afford 5-(5-hydroxy-1H-indole-3-yl)-3-(3,4,5-trime thoxyphenyl)pyrazin-2(1H)-one, compound 8. Yield: (52 mg, 64\%); mp $340{ }^{\circ} \mathrm{C} ;{ }^{1} \mathrm{H} \mathrm{NMR} \mathrm{(300} \mathrm{MHz,}$ DMSO-d $\left.d_{6}\right) \delta: 3.76$ (s, 3H, OMe), 3.88 (s, 6H, 2 OMe), 6.68 (dd, $\left.J=8.7,2.33 \mathrm{~Hz}, 1 \mathrm{H}, \mathrm{H}-6^{\prime \prime}\right), 7.23$ (d, $\left.J=8.6 \mathrm{~Hz}, 1 \mathrm{H}, \mathrm{H}-7^{\prime \prime}\right), 7.64$ (d, $\left.J=2.2 \mathrm{~Hz}, 1 \mathrm{H}, \mathrm{H}-44^{\prime \prime}\right), 7.79$ (s, 1H, H-6), 7.80 (d, $\left.J=2.7 \mathrm{~Hz}, 1 \mathrm{H}, \mathrm{H}-2^{\prime \prime}\right)$, 8.00 (s, 2H, H-2',6'), 8.67 (s, 1H, OH), 11.05 (d, $\left.J=2.4 \mathrm{~Hz}, 1 \mathrm{H}, \mathrm{NH}-1^{\prime \prime}\right), 12.47$ (s, 1H, NH-1); ${ }^{13} \mathrm{C}$ NMR (75 MHz, DMSO-d $\left.d_{6}\right)$ 8: 55.7 (2 OMe), 60.1 (OMe), 104.7 (CH-4"), $106.0\left(\mathrm{CH}-2^{\prime}, 6^{\prime}\right), 111.3\left(\mathrm{C}_{\mathrm{q}^{-}} 3^{\prime \prime}\right)$, 111.8, 111.9 (CH-6",7"), 123.5 (CH-2",6), $125.7\left(\mathrm{C}_{\mathrm{q}}-4^{\prime}\right), 131.9\left(\mathrm{C}_{\mathrm{q}}-1^{\prime \prime}, 7 \mathrm{a}^{\prime \prime}\right), 132.8\left(\mathrm{C}_{\mathrm{q}}-3 \mathrm{a}^{\prime \prime}\right), 138.8$ $\left(\mathrm{C}_{\mathrm{q}^{-}} 4^{\prime}\right), 151.2\left(\mathrm{COH}-5^{\prime \prime}\right), 152.3\left(\mathrm{C}_{\mathrm{q}^{-}} 3^{\prime}, 5^{\prime}\right), 154.0(\mathrm{CO}-2)$; LC-MS $m / z 394[\mathrm{M}+\mathrm{H}]^{+}$.

\subsection{Molecular Modeling}

All modeling was performed on a DELL 8 core system. For visualization and building the structures Maestro (version 9.3) from Schrödinger (Schrödinger, LLC, New York, NY, USA, 2012) was used (VEGF-R2 pdb code 2p2h, [23]). The illustrations of modeling were generated by Maestro. For compound docking and screening the Schrödinger "Glide SP" workflow was used [28]. The goal of the Glide methodology is to semiquantitatively rank the ability of candidate ligands to bind to a specified conformation of the protein receptor. Prior to determining binding poses of ligands energetically minimized compound conformations were generated, docked into the active site and subsequently ranked based on their calculated binding affinity.

\subsection{Biological Evaluation}

All inhibitor solutions were prepared freshly in DMSO prior to each experiment and used immediately.

\subsubsection{Selectivity Profiling of Compounds by $\mathrm{IC}_{50}$ Values Using 24 Protein Kinases}

Recombinant protein kinases. The inhibitory profile of compounds was determined using the following 24 protein kinases (GenBankAcc.No. available on http://www.proqinase.com/pages/science [29]): AKT1, ARK5, Aurora-A, Aurora-B, B-Raf-VE, CDK2/CycA, CDK4/CycD1, CK2-A1, EGF-R, EPHB4, ERBB2, FAK, IGF1-R, SRC, VEGF-R2, VEGF-R3, FLT3, INS-R, MET, PDGFRß, PLK1, SAK, TIE2 and COT. All protein kinases were expressed using human cDNAs in Sf9 insect cells as recombinat GST-fusion proteins or His-tagged proteins by means of the baculovirus expression system. Kinases were purified by affinity chromatography using either GSH-agarose (Sigma) or Ni-NTA-agarose (Qiagen). The purity and identity of each kinase was determined by SDS-PAGE/silver staining and western blot analysis using specific antibodies.

Protein kinase Assay. A proprietary protein kinase assay $\left({ }^{33}\right.$ PanQinase ${ }^{\circledR}$ Activity Assay) was used for measuring the kinase activity of the 24 protein kinases. All protein kinase assays were performed in 96-well FlashPlates ${ }^{\mathrm{TM}}$ (Perkin Elmer/NEN, Boston, MA, USA) in a $50 \mu \mathrm{L}$ reaction volumes. Assays for all enzymes were performed in a solution containing $60 \mathrm{mM} \mathrm{HEPES-NaOH}, \mathrm{pH} 7.5,3 \mathrm{mM} \mathrm{MgCl}$, 
$3 \mathrm{mM} \mathrm{MnCl}_{2}, 3 \mu \mathrm{M}$ Na-orthovanadate, $1.2 \mathrm{mM}$ DTT, $50 \mu \mathrm{g} / \mathrm{mL}$ PEG20000, $1 \mu \mathrm{M}[\gamma$-33P]-ATP (approx. $5 \times 105 \mathrm{cpm}$ per well), recombinant protein kinase (50-400 ng). Depending upon the kinase being assayed, appropriate substrates were used and were as follows (substrates shown in parentheses): AKT1 (GSK3/14-27), ARK5 (autophosphorylation), Aurora-A, Aurora-B (Tetra(LRRWSLG)), B-Raf-VE (MEK1 KM), CDK2/CycA (histone H1), CDK4/CycD1 (Rb-CTF), CK2-A1 (Casein), EGF-R, EPHB4, ERBB2, FAK, IGF1-R, SRC, VEGF-R2, VEGF-R3 (poly(Glu,Tyr) 4:1), FLT3, INS-R, MET, PDGFR $\beta$ (poly(Ala,Glu,Lys,Tyr) 6:2:5:1), PLK1 (Casein), SAK (autophosphorylation), TIE2 (poly(Glu,Tyr) 4:1), COT (autophosphorylation). The $\mathrm{IC}_{50}$ values were measured by testing 10 concentrations of compounds by single sampling. The final DMSO concentration in the assay was $1 \%(\mathrm{v} / \mathrm{v})$. The data were fitted using the 4-parameter logistic fit option of GraphPad Prism 5.

\subsubsection{Cell Culture and Proliferative Assays using HL-60, TK 10, 786-0, M 14, and MCF-7 Cells}

The cells were grown in RPMI 1640 Glutamax with $10 \%$ FCS, $100 \mu \mathrm{g} / \mathrm{mL}$ streptomycin and $100 \mathrm{U} / \mathrm{mL}$ Penicillin $\mathrm{G}$ and incubated in a $5 \% \mathrm{CO}_{2}$ humidified atmosphere at $37{ }^{\circ} \mathrm{C}$. For proliferation experiments, cells were seeded in $20 \mu \mathrm{L}$ pro well into 384 -well Greiner 384 CellStar $^{\circledR}$ plates (Greiner Bio-One I. AG, Kremsmünster, AT). In addition to the test plates, one plate was prepared for the reference measurement at day zero. All plates were incubated for $24 \mathrm{~h}$ at $37{ }^{\circ} \mathrm{C}$ in a humidified atmosphere with $5 \% \mathrm{CO}_{2}$. Compounds 5 and $\mathbf{8}$ that were dissolved in 100\% DMSO (v/v) were added to test plates using the Echo $550^{\circledR}$ Liquid Handler (Labcyte Inc., Sunnyvale, UK). The final DMSO concentration in the assay was $0.5 \% \mathrm{v} / \mathrm{v}$. The viability of the cells in the day zero control plates were determined on the same day without adding any compounds. The CellTiter-Glo ${ }^{\circledR}$ Viability Assay was used to determine the viability of cells using the standard protocol for this assay (Promega Corp., Madison, WI, USA). The luminescence signal was measured at the EnSpire ${ }^{\circledR}$ Multimode Plate Reader (PerkinElmer, Waltham, MA, USA). Test plates were incubated for further $48 \mathrm{~h}$ and the cell viability was defined as just described. Measured raw data were converted into percent of cell growth by using the high control $(0.5 \%$ DMSO v/v without compound) and the day zero control. For dose-response studies, 11 different concentrations of compounds were tested in quadruplicates. The $\mathrm{IC}_{50}$ values were calculated using the 4-parameter logistic fit option of GraphPad Prism 5.

\section{Conclusions}

In this study, we developed pyrazin-2(1H)-ones as potent and PDGFR $\beta$ inhibitors based on marine derived hamacanthins. Modeling studies showed the core moiety of hamacanthins to bind in the ATP binding pocket of RTK and suggested a straightforward strategy towards potent PDGFR $\beta$ binders. For subsequent optimization of hamacanthin derivatives as PDGFR $\beta$ inhibitors, a flexible synthetic route via microwave-mediated ring closure to asymmetric 3,5-substituted pyrazin-2(1H)-ones was established and a set of novel compounds was produced. Herein, we identified highly potent PDGFR $\beta$ binders with $\mathrm{IC}_{50}$ values in an enzymatic assay below $\mu \mathrm{M}$ range with compound $\mathbf{5}$ possessing significant activity against PDGFR dependent cancer cells. Thus, marine hamacanthin-derived pyrazin-2 $(1 H)$-ones showed interesting properties as lead for the further development of highly potent and selective PDGFR $\beta$-inhibitors. 


\section{Acknowledgments}

We thank Martin Schütt for excellent technical assistance during synthesis at the Institute of Pharmacy, Kiel and Janina Rahlff for excellent cell culture assistance at the European ScreeningPort $\mathrm{GmbH}$, Hamburg.

\section{Conflicts of Interest}

The authors declare no conflict of interest.

\section{References}

1. Haefner, B. Drugs from the deep: Marine natural products as drug candidates. Drug Discov. Today 2003, 8, 536-544.

2. Gupta, L.; Talwar, A.; Chauhan, P.M. Bis and tris indole alkaloids from marine organisms: New leads for drug discovery. Curr. Med. Chem. 2007, 14, 1789-1803.

3. Skropeta, D.; Pastro, N.; Zivanovic, A. Kinase inhibitors from marine sponges. Mar. Drugs 2011, 9, 2131-2154.

4. Kouko, T.; Matsumura, K.; Kawasaki, T. Total synthesis of marine bisindole alkaloids, (+)-hamacanthins A, B and (-)-antipode of cis-dihydrohamacanthin B. Tetrahedron 2005, 61, 2309-2318.

5. Bao, B.; Sun, Q.; Yao, X.; Hong, J.; Lee, C.-O.; Cho, H.Y.; Jung, J.H. Bisindole alkaloids of the topsentin and hamacanthin classes from a marine sponge Spongosorites sp. J. Nat. Prod. 2007, $70,2-8$.

6. Casapullo, A.; Bifulco, G.; Bruno, I.; Riccio, R. New bisindole alkaloids of the topsentin and hamacanthin classes from the Mediterranean marine sponge Rhaphisia lacazei. J. Nat. Prod. 2000, 63, 447-451.

7. Peifer, C.; Bühler, S.; Hauser, D.; Kinkel, K.; Totzke, F.; Schaechtele, C.; Laufer, S. Design, synthesis and characterization of N9/N7-substituted 6-aminopurines as VEGF-R and EGF-R inhibitors. Eur. J. Med. Chem. 2009, 44, 1788-1793.

8. Peifer, C.; Kinkel, K.; Abadleh, M.; Schollmeyer, D.; Laufer, S. From five- to six-membered rings: 3,4-diarylquinolinone as lead for novel p38MAP kinase inhibitors. J. Med. Chem. 2007, 50, 1213-1221.

9. Peifer, C.; Krasowski, A.; Hämmerle, N.; Kohlbacher, O.; Dannhardt, G.; Totzke, F.; Schaechtele, C.; Laufer, S. Profile and molecular modeling of 3-(indole-3-yl)-4-(3,4,5-trimethoxyphenyl)-1Hpyrrole-2,5-dione(1) as a highly selective VEGF-R2/3 inhibitor. J. Med. Chem. 2006, 49, 7549-7553.

10. Peifer, C.; Selig, R.; Kinkel, K.; Ott, D.; Totzke, F.; Schaechtele, C.; Heidenreich, R.; Röcken, M.; Schollmeyer, D.; Laufer, S. Design, synthesis, and biological evaluation of novel 3-aryl-4-(1H-indole-3yl)-1,5-dihydro-2H-pyrrole-2-ones as vascular endothelial growth factor receptor (VEGF-R) inhibitors. J. Med. Chem. 2008, 51, 3814-3824.

11. Jiang, B.; Yang, C.G.; Wang, J. Enantioselective synthesis for the (-)-antipode of the pyrazinone marine alkaloid, hamacanthin A. J. Org. Chem. 2001, 66, 4865-4869. 
12. Miyake, F.Y.; Yakushijin, K.; Horne, D.A. Synthesis of marine sponge bisindole alkaloids dihydrohamacanthins. Org. Lett. 2002, 4, 941-943.

13. Zoraghi, R.; Worrall, L.; See, R.H.; Strangman, W.; Popplewell, W.L.; Gong, H.; Samaai, T.; Swayze, R.D.; Kaur, S.; Vuckovic, M.; et al. Methicillin-resistant Staphylococcus aureus (MRSA) pyruvate kinase as a target for bis-indole alkaloids with antibacterial activities. J. Biol. Chem. 2011, 286, 44716-44725.

14. Caldwell, J.J.; Veillard, N.; Collins, I. Design and synthesis of 2(1H)-pyrazinones as inhibitors of protein kinases. Tetrahedron 2012, 68, 9713-9728.

15. Dar, A.C.; Shokat, K.M. The evolution of protein kinase inhibitors from antagonists to agonists of cellular signaling. Ann. Rev. Biochem. 2011, 80, 769-795.

16. Appelmann, I.; Liersch, R.; Kessler, T.; Mesters, R.; Berdel, W. Angiogenesis inhibition in cancer therapy: Platelet-derived growth factor (PDGF) and vascular endothelial growth factor (VEGF) and their receptors: Biological functions and role in malignancy. Recent Results Cancer Res. 2010, 180, 51-81.

17. Koch, S.; Tugues, S.; Li, X.; Gualandi, L.; Claesson-Welsh, L. Signal transduction by vascular endothelial growth factor receptors. Biochem. J. 2011, 437, 169-183.

18. Zhou, Y.; Chen, Y.; Tong, L.; Xie, H.; Wen, W.; Zhang, J.; Xi, Y.; Shen, Y.; Geng, M.; Wang, Y.; et al. AL3810, a multi-tyrosine kinase inhibitor, exhibits potent anti-angiogenic and anti-tumour activity via targeting VEGFR, FGFR and PDGFR. J. Cell. Mol. Med. 2012, 16, 2321-2330.

19. Noble, M.E.M.; Endicott, J.A.; Johnson, L.N. Protein kinase inhibitors: Insights into drug design from structure. Science 2004, 303, 1800-1805.

20. Norman, R.A.; Toader, D.; Ferguson, A.D. Structural approaches to obtain kinase selectivity. Trends Pharmacol. Sci. 2012, 33, 273-278.

21. Sachsenmaier, C.; Schachtele, C. Integrated technology platform protein kinases for drug development in oncology. Biotechniques 2002, 33, S101-S106.

22. Heldin, C.H.; Westermark, B. Mechanism of action and in vivo role of platelet-derived growth factor. Physiol. Rev. 1999, 79, 1283-1316.

23. Hodous, B.L.; Geuns-Meyer, S.; Hughes, P.; Albrecht, B.; Bellon, S.; Bready, J.; Caenepeel, S.; Cee, V.; Chaffee, S.; Coxon, A.; et al. Evolution of a highly selective and potent 2-(pyridin-2-yl)-1, 3,5-triazine tie-2 kinase inhibitor. J. Med. Chem. 2007, 50, 611-626.

24. Garuti, L.; Roberti, M.; Bottegoni, G. Non-ATP competitive protein kinase inhibitors. Curr. Med. Chem. 2010, 17, 2804-2821.

25. Peifer, C.; Stoiber, T.; Unger, E.; Totzke, F.; Schaechtele, C.; Marmé, D.; Brenk, R.; Klebe, G.; Schollmeyer, D.; Dannhardt, G. Design, synthesis, and biological evaluation of 3,4-diarylmaleimides as angiogenesis inhibitors. J. Med. Chem. 2006, 49, 1271-1281.

26. Reiterer, G.; Yen, A. Platelet-derived growth factor receptor regulates myeloid and monocytic differentiation of HL-60 cells. Cancer Res. 2007, 67, 7765-7772.

27. Johannes, E.; Horbert, R.; Schlosser, J.; Schmidt, D.; Peifer, C. Effective synthesis of 3,5-diaryl-(1H)-pyrazin-2-ones via microwave mediated ring closure. Tetrahedron Lett. 2013, 54, 4067-4072. 
28. Halgren, T.A.; Murphy, R.B.; Friesner, R.A.; Beard, H.S.; Frye, L.L.; Pollard, W.T.; Banks, J.L. Glide: A new approach for rapid, accurate docking and scoring. 2. Enrichment factors in database screening. J. Med. Chem. 2004, 47, 1750-1759.

29. ProQinase Targeting Cancer. Available online: http://www.proqinase.com (accessed on 19 August 2013).

(C) 2013 by the authors; licensee MDPI, Basel, Switzerland. This article is an open access article distributed under the terms and conditions of the Creative Commons Attribution license (http://creativecommons.org/licenses/by/3.0/). 